\title{
BMJ Open Implications of parental lifestyle changes and education level on adolescent offspring weight: a population based cohort study - The HUNT Study, Norway
}

Marit Naess, ${ }^{1,2}$ Erik R Sund, ${ }^{1,3}$ Turid Lingaas Holmen, ${ }^{1}$ Kirsti Kvaløy ${ }^{1,2}$

To cite: Naess M, Sund ER, Holmen TL, et al. Implications of parental lifestyle changes and education level on adolescent offspring weight: a population based cohort study - The HUNT Study, Norway. BMJ Open 2018;8:e023406. doi:10.1136/ bmjopen-2018-023406

- Prepublication history and additional material for this paper are available online. To view these files, please visit the journal online (http://dx.doi. org/10.1136/bmjopen-2018023406).

Received 5 April 2018 Revised 26 June 2018 Accepted 31 July 2018

Check for updates

(c) Author(s) (or their employer(s)) 2018. Re-use permitted under CC BY-NC. No commercial re-use. See rights and permissions. Published by BMJ.

${ }^{1}$ Department of Public Health and Nursing, Faculty of Medicine and Health Sciences, HUNT Research Centre, NTNU Norwegian University of Science and Technology, Trondheim,

Norway

${ }^{2}$ Department of Research and Development, Levanger Hospital, Nord-Trøndelag Health Trust, Levanger, Norway

${ }^{3}$ Faculty of Nursing and Health Sciences, Nord University, Levanger, Norway

Correspondence to

Marit Naess;

marit.nass@ntnu.no

\section{ABSTRACT}

Objective Obesity tends to cluster in families reflecting both common genetics and shared lifestyle patterns within the family environment. The aim of this study was to examine whether parental lifestyle changes over time, exemplified by changes in weight and physical activity, could affect offspring weight in adolescents and if parental education level influenced the relationship.

Design, setting and participants The population-based cohort study included 4424 parent-offspring participants from the Nord-Trøndelag Health Study, Norway. Exposition was parental change in weight and physical activity over 11 years, and outcome was offspring weight measured in z-scores of body mass index (BMI) in mixed linear models. Results Maternal weight reduction by $2-6 \mathrm{~kg}$ was significantly associated with lower offspring BMl z-scores: $-0.132(95 \% \mathrm{Cl}-0.259$ to -0.004$)$ in the model adjusted for education. Parental weight change displayed similar effect patterns on offspring weight regardless of parents' education level. Further, BMI was consistently lower in families of high education compared with low education in the fully adjusted models. In mothers, reduced physical activity level over time was associated with higher BMI z-scores in offspring: 0.159 (95\% $\mathrm{Cl} 0.030$ to 0.288$)$. Associations between physical activity change and adolescent BMI was not moderated by parental education levels.

Conclusion Lifestyle changes in mothers were associated with offspring BMI; reduced weight with lower-and reduced physical activity with higher BMI. Father's lifestyle changes, however, did not significantly affect adolescent offspring's weight. Overall, patterns of association between parental changes and offspring's BMI were independent of parental education levels, though adolescents with parents with high education had lower weight in general.

\section{INTRODUCTION}

Despite the past years' enhanced efforts investigating causes and mechanisms of the rapid rise in obesity, there are still numerous aspects that are poorly understood. The major cause of obesity is the imbalance between energy consumption and energy expenditure where
Strengths and limitations of this study

- This study has a large number of parent-offspring participants, and use the same protocol for anthropometric measurements in parents and offspring.

- The large number of mother-offspring duos $(n=3770)$ enabled the significant associations between mothers lifestyle changes and offspring weight to be identified.

- Trained health personnel using standardised methods carried out the anthropometric measurements.

- The study has only addressed the potential effects of a two obesogenic factors (weight and physical activity), although these are among the more important ones.

- The reliability and validity of the self-reported physical activity levels may be a limitation in our study.

both genetics and environmental factors play an important role. ${ }^{1-3}$ Since the genetic predisposition has not changed during the past few decades, however, changes in environmental exposures are the main drivers behind the obesity increase. ${ }^{4}$ Physical activity, sedentary behaviour and healthy eating are all important factors influencing obesity, where also parental lifestyle seems to be highly correlated with offspring's lifestyle. ${ }^{56}$ Additionally, the time frame being exposed to a more obesogenic environment seems to be of great importance concerning weight gain in the population. ${ }^{78}$

Adult behaviours may be guided by conscious choices, but are also strongly dependent on socioeconomic status (SES) and the influence of surrounding local environment. ${ }^{9}$ Low SES is a well-known obesity risk factor $^{10} 11$ where especially differences in diet may reflect economic income. Differential offspring weight linked to family SES is however also dependent on whether parents 
are lean or not. ${ }^{12}$ In low SES families with obese parents, offspring are of higher weight. Changes in family lifestyle influence the body mass index (BMI) of both parents and offspring, which support the notion that shared environment is of great concern with regard to offspring obesity development. ${ }^{13}$ Parental prepregnancy obesity influence offspring weight ${ }^{14}$ and a previous study from Norway found that children of parents who went from normal weight to overweight during 11 years had a higher risk of overweight as adolescents. ${ }^{15}$ Likewise, family weight loss programmes have shown that reduction in parental weight positively influence offspring weight. ${ }^{1617}$

Both in adults, adolescents and children, the potential effects on one's weight due to changes in behaviours related to known obesogenic factors such as physical activity behaviour and nutrition, have been studied. ${ }^{4} 18$ Previous studies have shown strong correlations between weight increase in parents and overweight and obesity in offspring. ${ }^{14} 19-21$ Few studies have however focused on the influence of parental lifestyle changes over time with offspring weight in population-based samples. The aim of this study was therefore to assess the impact of parental changes in weight and physical activity on offspring weight at adolescence and if these relationships were differentially influenced by parental education level. We hypothesised that decrease in parental weight and increase in physical activity over time will be associated with lower offspring weight.

\section{MATERIALS AND METHODS}

\section{Study sample}

The Nord-Trøndelag Health Study (HUNT) ${ }^{22-24}$ is a large population-based cohort study conducted in the middle of Norway, covering 125000 participants aged 13 years and above. It consists of three health surveys taken place in 1984-1986 (HUNT1), 1995-1997 (HUNT2 and YoungHUNT1) and 2006-2008 (HUNT3 and Young-HUNT3). Data were collected in all 24 municipalities of the county, and the health examinations were performed by professional health staff in temporarily located sites. ${ }^{24}$ The Young-HUNT Study was conducted in all junior and senior high schools in the county as the adolescent part (13-19 years) of the HUNT Study. The Young-HUNT3 Survey included 7716 participants who had completed both the questionnaire and clinical examination (response rate $74 \%$ ). Adolescents were linked to their parents through a common ID.

In our study, 4424 adolescents (2201 girls/2223 boys) had parents (3770 mothers and 2985 fathers) who had participated in both HUNT2 (1995-1997) and at the follow-up HUNT3 (2006-2008) (mean follow-up period was 11 years), in addition to having data available on the variables to be included in the study (figure 1). Due to missing information on parents' reported physical activity at follow-up (HUNT3), only 2997 mothers and 2248 fathers were included in the analyses related to physical activity change. The lower response rate on the physical activity questions in HUNT3 compared with HUNT2 was due to the different placing of the questions which impacted the response rate. ${ }^{24}$

This protocol is in accordance with the Declaration of Helsinki.

\section{Patient and public involvement}

This was a population-based study, hence no patients or public were involved.

\section{Measurements}

Data collection included self-reported questionnaires and clinical measurements. ${ }^{24}$ Trained nurses performed the measurements of height and weight at the screening

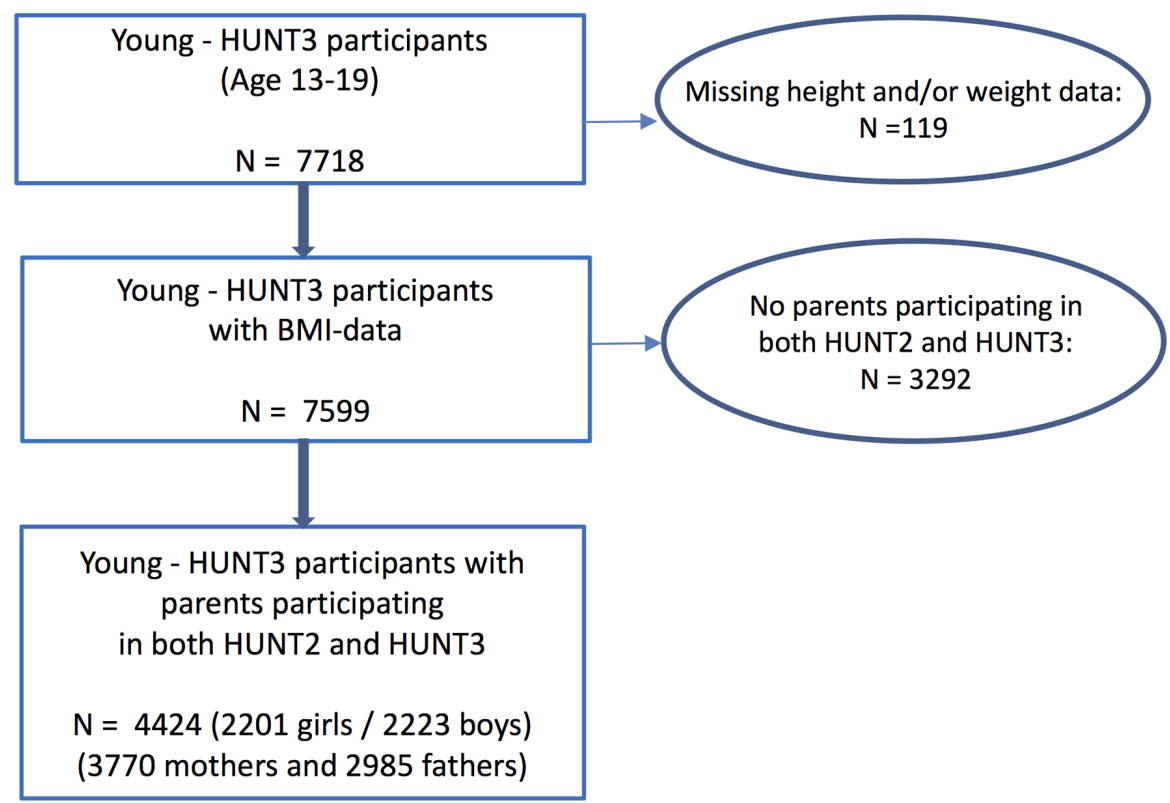

Figure 1 Study flow chart. BMI, body mass index; HUNT, Nord-Trøndelag Health Study. 
Table 1 Descriptive characteristics* of offspring and parents

\begin{tabular}{|c|c|c|c|c|c|c|}
\hline & \multirow{2}{*}{$\frac{\text { Daughters }}{2006-2008}$} & \multirow{2}{*}{$\begin{array}{l}\text { Sons } \\
2006-2008\end{array}$} & \multicolumn{2}{|l|}{ Mothers } & \multicolumn{2}{|l|}{ Fathers } \\
\hline & & & $1995-1997$ & 2006-2008 & 1995-1997 & 2006-2008 \\
\hline No of participants & 2201 & 2223 & 3770 & 3770 & 2985 & 2985 \\
\hline Height, cm (SD) & $165.1(6.4)$ & $174.3(9.4)$ & $166.6(5.9)$ & $166.6(6.0)$ & $180.0(6.3)$ & $179.5(6.2)$ \\
\hline Weight, kg (SD) & $60.5(11.2)$ & $67.5(14.8)$ & $69.2(11.9)$ & $73.8(13.5)$ & $84.4(11.6)$ & $89.0(12.8)$ \\
\hline Overweight†, n (\%) & $392(18)$ & $413(19)$ & $1143(30)$ & $1437(38)$ & $1502(50)$ & $1669(56)$ \\
\hline Obese†, n (\%) & $92(4)$ & $131(6)$ & $384(10)$ & $736(20)$ & $284(10)$ & $656(22)$ \\
\hline BMI z-score (SD) $\ddagger$ & $-0.021(0.97)$ & $-0.029(0.96)$ & & & & \\
\hline \multicolumn{7}{|l|}{ Parents education level§ } \\
\hline \multicolumn{7}{|l|}{ Physical activity } \\
\hline Hard, n (\%) & & & $183(6.1)$ & $604(20.1)$ & $307(13.7)$ & $429(19.1)$ \\
\hline Moderate, n (\%) & & & $1284(42.8)$ & 1466 (48.9) & $881(39.2)$ & $976(43.4)$ \\
\hline Low, n (\%) & & & 1057 (35.3) & $719(24.0)$ & $699(31.1)$ & $638(28.4)$ \\
\hline Inactive, n (\%) & & & $473(15.8)$ & $208(6.9)$ & $361(16.1)$ & $205(9.1)$ \\
\hline
\end{tabular}

${ }^{*}$ Data presented as mean with SD, unless otherwise specified.

†BMI (body mass index) categories in adolescents are age and sex adjusted in accordance with Cole et al. ${ }^{26}$ $\ddagger$ BMI z-score mean values are based on the whole Young-HUNT3 population.

$\S L o w=0-10$ years school attendance, medium $=11-14$ years school attendance, high $>14$ years school attendance, Statistics Norway. ${ }^{29}$

ๆHard $\geq 3$ hours hard activity/week, moderate $\geq 3$ hours light activity and/or 1-2 hours hard activity/week, low=1-2 hours light activity and/or $<1$ hour hard activity/week, Inactive $\leq 1$ hour light activity and no hard activity/week.

HUNT, Nord-Trøndelag Health Study.

stations for the adults and at the schools for the adolescents. Light clothes and no shoes were allowed during the measurements, and internally standardised meters and weight scales were used. ${ }^{23}$ We estimated the BMI from weight in kilograms divided by squared height in metres $\left(\mathrm{kg} / \mathrm{m}^{2}\right)$.

Weight categories in adults (table 1) were defined according to WHO; underweight as BMI $<18.5 \mathrm{~kg} / \mathrm{m}^{2}$, normal weight as BMI $18.5-24.9 \mathrm{~kg} / \mathrm{m}^{2}$, overweight as BMI $25-29.9 \mathrm{~kg} / \mathrm{m}^{2}$ and obesity as BMI $\geq 30 \mathrm{~kg} / \mathrm{m}^{2}{ }^{25}$ As there were only 44 and 21 underweight mothers (HUNT2 and HUNT3, respectively) and 5 and 4 underweight fathers (HUNT2 and HUNT3, respectively), we combined the underweight and normal weight categories in the respective surveys. The adolescent BMI-based weight categories were defined using the age-specified and sex-specified International Obesity Task Force cut-off values. ${ }^{26}$ Adolescent BMI measurements were also transformed into age-specific and sex-specific z-score values where z-scores indicate the SD of the BMI measure above (positive values) or below (negative values) the expected mean of the reference population (Young-HUNT3). Age was defined as the nearest birthday, for example, age 14 years included $\geq 13.5$ and $<14.5$ years of age.

Both in HUNT2 and HUNT3 participants were asked the following question concerning physical activity: 'How has your physical activity in leisure time been during the last year?' The answering options related to frequency were: 'none', 'less than 1 hour a week', '1-2 hours a week' and ' 3 or more hours a week. They also were told to estimate the number of hours of light (no sweat/not being out of breath) - and hard (sweat/out of breath) activity per week. Activity level, a combination of frequency answers and options from the 'hard' and 'light' classification were further divided into four: hard ( $\geq 3$ hours hard activity/week), moderate ( $\geq 3$ hours light activity and/ or 1-2 hours hard activity/week), low (1-2 hours light activity and/or $<1$ hour hard activity/week) and inactive ( $\leq 1$ hour light activity and no hard activity/week). The 'light' physical activity category has previously been shown to be in adequate correlation with the moderate intensity physical activity measure from the International Physical Activity Questionnaire and the 'hard' physical activity category to be a valid measure of vigorous intensity compared with previous studies. ${ }^{27} 28$ 
Data on parental changes in weight and physical activity were ascertained at the two time points HUNT2 (1995-1997) and HUNT3 (2006-2008). The following five weight change categories were chosen based on previous literature ${ }^{15}$ : (1) more than $6 \mathrm{~kg}$ weight increase, (2) $2-6 \mathrm{~kg}$ weight increase, (3) no weight change $\pm 0-2 \mathrm{~kg}$, (4) $2-6 \mathrm{~kg}$ weight reduction and (5) more than $6 \mathrm{~kg}$ weight reduction. We classified parental change in physical activity as: (1) increased activity (one or more activity levels up), (2) no change and (3) decreased activity (one or more activity levels down).

Estimation of parental SES was done by using their education level divided into three categories based on the Norwegian Standard Classification of Education: low $=0-10$ years of school attendance, medium: 11-14 years of school attendance and high: $>14$ years of school attendance. ${ }^{29}$ In the initial analyses, we found effect estimates for low and medium education to be similar,

Table 2 Associations between parental weight change and offspring BMI z-scores

\begin{tabular}{|c|c|c|c|c|c|c|c|c|}
\hline & \multicolumn{2}{|c|}{ Model 1} & \multicolumn{2}{|c|}{ Model 2} & \multicolumn{2}{|c|}{ Model 3} & \multicolumn{2}{|c|}{ Model 4} \\
\hline & b & $95 \% \mathrm{Cl}$ & b & $95 \% \mathrm{Cl}$ & b & $95 \% \mathrm{Cl}$ & b & $95 \% \mathrm{Cl}$ \\
\hline \multicolumn{9}{|l|}{ Maternal } \\
\hline \multicolumn{9}{|l|}{ Weight change (WeC) } \\
\hline Increased $>6 \mathrm{~kg}$ & & & 0.063 & -0.020 to 0.146 & 0.050 & -0.032 to 0.133 & 0.030 & -0.078 to 0.137 \\
\hline Reduced 2-6 kg & & & -0.124 & -0.251 to 0.004 & -0.132 & -0.259 to -0.004 & -0.106 & -0.270 to 0.058 \\
\hline Reduced $>6 \mathrm{~kg}$ & & & -0.087 & -0.255 to 0.081 & -0.103 & -0.271 to 0.064 & -0.011 & -0.211 to 0.189 \\
\hline \multicolumn{9}{|l|}{ Education } \\
\hline Low & & & & & 0 & (Ref) & 0 & (Ref) \\
\hline \multicolumn{9}{|l|}{ Interaction WeCxeducation } \\
\hline Increased 2-6 kg×high & & & & & & & -0.036 & -0.212 to 0.140 \\
\hline Reduced 2-6kg×high & & & & & & & -0.066 & -0.325 to 0.193 \\
\hline Reduced $>6 \mathrm{~kg} \times$ high & & & & & & & -0.324 & -0.680 to 0.031 \\
\hline \multicolumn{9}{|l|}{ Paternal } \\
\hline Baseline BMI & 0.076 & 0.065 to 0.088 & 0.077 & 0.066 to 0.089 & 0.076 & 0.065 to 0.087 & 0.076 & 0.065 to 0.087 \\
\hline \multicolumn{9}{|l|}{ WeC } \\
\hline Increased $>6 \mathrm{~kg}$ & & & 0.062 & -0.030 to 0.154 & 0.050 & -0.042 to 0.142 & 0.065 & -0.041 to 0.172 \\
\hline Increased 2-6 kg & & & -0.003 & -0.101 to 0.095 & -0.011 & -0.108 to 0.087 & -0.024 & -0.136 to 0.089 \\
\hline Stable & & & 0 & (Ref) & 0 & (Ref) & 0 & (Ref) \\
\hline \multicolumn{9}{|l|}{ Interaction WeCxeducation } \\
\hline Increased $>6 \mathrm{~kg} \times$ high & & & & & & & -0.062 & -0.272 to 0.148 \\
\hline Increased 2-6 kg×high & & & & & & & 0.060 & -0.163 to 0.282 \\
\hline Reduced 2-6kg×high & & & & & & & -0.232 & -0.550 to 0.097 \\
\hline Reduced $>6 \mathrm{~kg} \times$ high & & & & & & & 0.196 & -0.383 to 0.777 \\
\hline
\end{tabular}

The numbers given are the linear mixed-effects regression coefficients between the exposure variables and covariates given as row names and the (age adjusted) BMI z-score of the offspring.

Model 1: Baseline BMI.

Model 2: Baseline BMI and adjustment for parents weight change.

Model 3: As model 2, but with further adjustment for education level.

Model 4: As model 3, but including the interaction term: weight changexeducation.

$\mathrm{BMI}$, body mass index. 

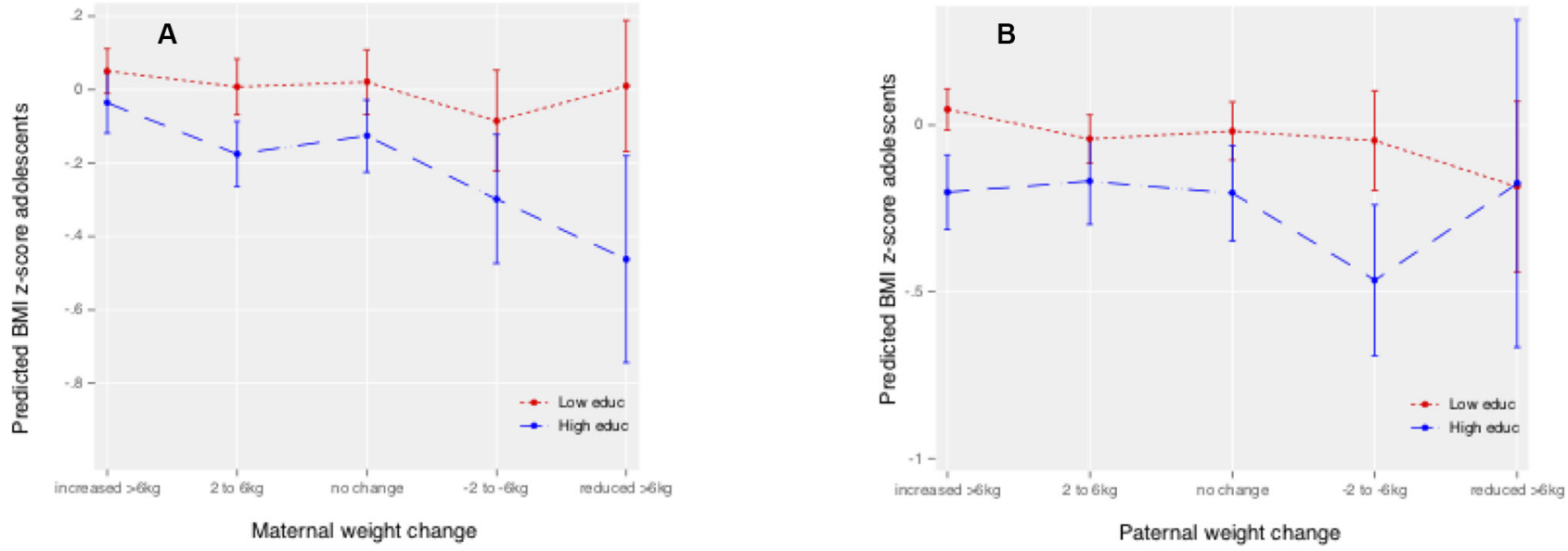

Figure 2 Predicted BMI z-scores in adolescent offspring related to maternal (A) and paternal (B) weight changes. BMI, body mass index.

thus, we collapsed these into two categories: low: $\leq 14$ years and high: $>14$ years. Data on education were obtained from Statistics Norway in $2010^{29}$ and linked through unique national identity numbers.

\section{Statistical analyses}

Data had a hierarchical structure with siblings nested within mothers or fathers in the respective analyses. Clustered on mothers ID, about $65 \%$ of the families in our study participated with one child. Furthermore, $35 \%$ of the families had siblings within the study sample with $32 \%$ consisting of two siblings and 3\% of three siblings or more. To account for this non-independence/clustering, we specified linear mixed-effects models. ${ }^{30}$ Models were built sequentially with increasing complexity by first adjusting for parental BMI at baseline in 1995-1997 (model 1) before the main predictors of interest (parental change in weight or physical activity) were added in model 2. In model 3 , we additionally added parental education and model 4 also included an interaction term between our main predictors of interest and education. We stratified our models by parental sex and predictor of interest (change in weight or physical activity). Fixed effects are reported as unstandardised beta coefficients with $95 \%$ CIs. All statistical analyses were conducted in Stata IC/V.14.2 (Stata Corporation).

\section{RESULTS}

\section{Descriptive statistics}

Subject characteristics are summarised in table 1 . Similar proportions of boys' and girls' families took part in the study. Most of the parents represented a medium education level corresponding to 11-14 years school attendance, but the proportion of mothers with a high education level were more than 1.5 times that of fathers. In both mothers and fathers, mean weight and BMI had increased about 6\% from 1995-1997 to 2006-2008, fewer individuals were normal weight and the number of obese individuals had doubled in the same period. The number of parents exerting hard and medium levels of physical activity had both increased, while physical inactivity had halved in both mothers and fathers during this period.

\section{Association between parental weight change and offspring BMI z-scores}

Concerning the associations between mothers' weight change and adolescent BMI z-scores, the only significant result was observed when mother's weight was reduced by $2-6 \mathrm{~kg}$ and education level was taken into account (adolescent BMI z-score: -0.132 , 95\% CI -0.259 to -0.004 , model 3) (table 2). Generally, high parental education levels were associated with lower adolescent BMI z-scores in both the maternal $(-0.152$, $95 \%$ CI -0.215 to -0.089$)$ and paternal $(-0.209,95 \%$ CI -0.288 to -0.130 ) analyses (model 3 , table 2 ). Model 4 , which included an interaction term between weight change and education, showed that the effect of weight change was similar in both education groups. (Likelihood ratio test, $\mathrm{p}>0.05$ ).

In contrast to the mother-offspring findings, there were no significant associations identified between father's weight change and adolescent offspring BMI z-scores. Results of the analyses in the fully adjusted models (model 4) are presented graphically in figure 2.

The trends of parental weight change effects on offspring BMI were the same regardless of parents' education level. BMI in offspring was generally higher at lower parental education levels. However, within both education groups, only a small proportion of parents had reduced their weight by more than $6 \mathrm{~kg}$ over time; $3 \%$ and $2 \%$ in the high education group and $5 \%$ and $2 \%$ in the low education group, mothers and fathers, respectively. Hence, results including these weight change categories must be interpreted with caution. The results of analyses, including only obese or overweight parents, showed the same patterns as those including all weight categories, although offspring BMI z-scores were generally higher (online supplementary figure S1a,b). 
Table 3 Associations between parental change in physical activity and offspring BMI z-scores

\begin{tabular}{|c|c|c|c|c|c|c|c|c|}
\hline & \multicolumn{2}{|c|}{ Model 1} & \multicolumn{2}{|c|}{ Model 2} & \multicolumn{2}{|c|}{ Model 3} & \multicolumn{2}{|l|}{ Model 4} \\
\hline & b & $95 \% \mathrm{Cl}$ & b & $95 \% \mathrm{Cl}$ & b & $95 \% \mathrm{Cl}$ & b & $95 \% \mathrm{Cl}$ \\
\hline \multicolumn{9}{|l|}{ Maternal } \\
\hline \multicolumn{9}{|l|}{ Change in PA (cPA) } \\
\hline Reduced & & & 0.139 & 0.035 to 0.243 & 0.125 & 0.021 to 0.229 & 0.159 & 0.030 to 0.288 \\
\hline \multicolumn{9}{|l|}{ Education } \\
\hline Low & & & & & 0 & (Ref) & 0 & (Ref) \\
\hline High & & & & & -0.153 & -0.223 to -0.082 & -0.117 & -0.231 to -0.002 \\
\hline \multicolumn{9}{|c|}{ Interaction cPAxeducation } \\
\hline Baseline BMI & 0.075 & 0.062 to 0087 & 0.074 & 0.062 to 0.088 & 0.074 & 0.060 to 0.085 & 0.073 & 0.060 to 0.085 \\
\hline \multicolumn{9}{|l|}{ cPA } \\
\hline Reduced & & & 0.063 & -0.040 to 0.163 & 0.043 & -0.060 to 0.145 & 0.083 & -0.032 to 0.199 \\
\hline Stable & & & 0 & (Ref) & 0 & (Ref) & 0 & (Ref) \\
\hline Increased & & & 0.035 & -0.053 to 0.122 & 0.033 & -0.054 to 0.120 & 0.047 & -0.055 to 0.149 \\
\hline \multicolumn{9}{|l|}{ Education } \\
\hline Low & & & & & 0 & (Ref) & 0 & (Ref) \\
\hline High & & & & & -0.219 & -0.307 to -0.131 & -0.166 & -0.299 to -0.033 \\
\hline \multicolumn{9}{|c|}{ Interaction cPAxeducation } \\
\hline
\end{tabular}

The numbers given are the linear mixed-effects regression coefficients between the exposure variables and covariates given as row names and the (age adjusted) BMI z-score of the offspring.

Model 1: Baseline BMI.

Model 2: Baseline BMl and adjustment for parents change in physical activity.

Model 3: As model 2, but with further adjustment for education level.

Model 4: As model 3, but including the interaction terms change in physical activityxeducation.

BMI, body mass index.

\section{Association between parental change in physical activity and adolescent offspring BMI z-scores}

The associations between parental physical activity change and adolescent offspring BMI z-scores are shown in table 3. Adjustment for mothers' baseline BMI in the analyses resulted in a significantly higher adolescent BMI z-score: 0.139 (95\% CI 0.035 to 0.243 ) when mothers' activity was reduced over time (model 2). Adjusting for mothers' education did not alter this effect (model 3), although a high education level was associated with a lower offspring adolescent BMI z-score $(-0.153,95 \%$ CI -0.223 to -0.082$)$. Specifying an interaction term between change in physical activity and education did not improve model fit (likelihood ratio test, $\mathrm{p}>0.05$ ) (model 4). Results of the analyses in the fully adjusted model are visually presented in figure $3 \mathrm{~A}$.
No significant associations were observed between fathers' change in physical activity and adolescent offspring BMI z-scores in any of the models. A high education level, however, was associated with a lower adolescent BMI z-score $(-0.219,95 \%$ CI -0.307 to -0.131$)$. Model 4 , where the interaction term between change in physical activity and education were taken into account, did not improve model fit (likelihood ratio test, $\mathrm{p}>0.05$ ). Results of the analyses in the fully adjusted model are visually presented in figure 3B.

\section{DISCUSSION}

In the present study, we examined how parental changes in weight and physical activity levels through their offspring's childhood, affected offspring BMI at adolescence. The principal findings of the study was that reduced weight 

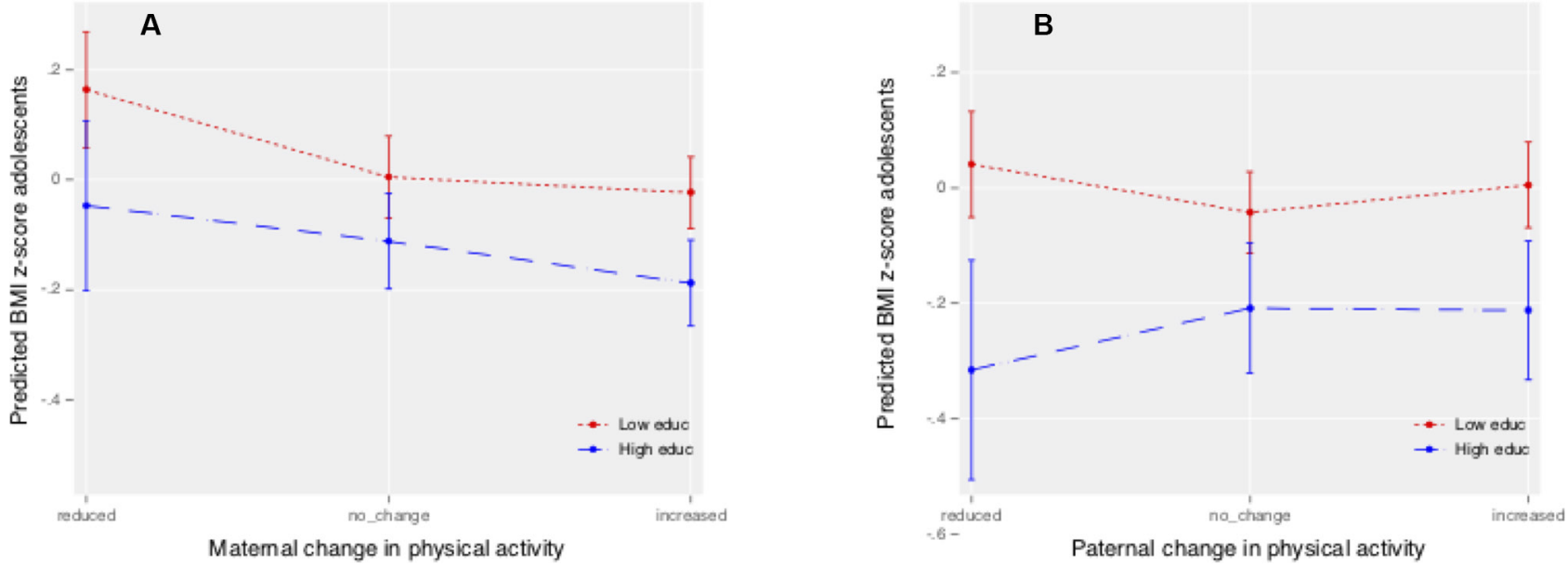

Figure 3 Predicted BMI z-score in adolescents offspring related to maternal $(A)$ and paternal $(B)$ changes in physical activity. BMI, body mass index.

in mothers was associated with lower offspring weight, while reduced physical activity was associated with higher offspring weight. Although not statistically significant, fathers' lifestyle changes showed the same direction of association. In agreement with other studies, we showed that parents' education level had an effect on adolescent offspring's weight. ${ }^{11} 1231$ The effects of parental changes in weight and level of physical activity, however, followed similar patterns regardless of parental education levels although stronger effects were observed in offspring with higher parental education.

Both genetic inheritance and social environment affect overweight and obesity. ${ }^{6} 1332$ Parents have a strong impact on their children's health ${ }^{33}$ where lifestyle and behavioural traits promoting obesity easily transmit from parents to children through family socialisation processes. Shared family environment means exposure to common obesogenic factors, such as unhealthy eating and a sedentary lifestyle. ${ }^{34}$ A systematic examination of the association between parental and child obesity across countries showed that children with overweight or obese parents are twice as likely to be overweight or obese as were their normal weight counterparts. ${ }^{35}$ Additionally, having two overweight or obese parents is more unfavourable than just having one. ${ }^{20}{ }^{35}$ There is no conclusive evidence that parents contribute equally to the risk of childhood overweight or obesity, ${ }^{35}$ although some studies suggest the maternal impact to be greater than the paternal. An Indian family study found only the maternal weight to be associated with child weight. ${ }^{36}$ Likewise, a Finnish study of 4788 mother-father-child trios found that maternal weight gain $\geq 8 \mathrm{~kg}$ was a significant predictor of adolescent overweight in both offspring genders. ${ }^{14}$ In the same study, paternal weight gain was only a significant predictor of adolescent overweight at higher levels $(\geq 18 \mathrm{~kg})$ and then only in daughters. ${ }^{14}$ A previous HUNT study showed that both parents' weight increase (from 1984-1986 to 19951997) was associated with a higher odds of overweight in adolescent offspring. ${ }^{15}$ In our study, a significant association between weight gain and offspring weight was only present between mothers and offspring. Although no significant association was observed between fathers and offspring, the parental BMI change pattern was similar in the parents. This discrepancy could be due to lower number of father-child duos compared with motherchild duos. By not including both mothers and fathers in the same models, we got a higher power in numbers of participants. Still, we cannot preclude that investigating full trios (mother-father-child) would not change the outcome estimates.

The importance of parents' physical activity and leisure-time behaviour related to offspring obesity development is well documented. ${ }^{6}$ In our study, we showed a general trend of a physical activity change from inactivity and low activity to moderate and hard activity over time in both mothers and fathers. Even so, this healthy tendency did not seem in general to have counteracted the increase in obesity over time. This finding may seem puzzling, but along with increased leisure time activity there is a simultaneous trend where large parts of the working hours are spent sitting and inactive. ${ }^{37} 38$ Thus, the overall activity level may have become increasingly unfavourable.

Nevertheless, the reported decrease in mothers' activity was associated to higher offspring BMI and this pattern seemed to be independent of education level. A previous study found both mother and father's activity and dietary patterns to predict girls risk of obesity. ${ }^{39}$ Thus, we could have expected changes in father's physical activity also to influence offspring weight. Although not statistically significant, our results did agree with the mentioned findings as increased paternal physical activity seemed to influence BMI in daughters more positively than in sons.

The grade of children's autonomy regarding the factors that influence weight is likely to be age dependent. Effects related to home diet and physical activity at school are expected to decrease by age. Therefore, one could expect the parental lifestyle changes to matter less in late adolescence compared with early adolescence. However, our results did not support differential age effects when 
models were tested separately in these two age groups, 13-15 years and 16-19 years of age (data not shown).

Our study confirmed the same overall pattern found in other studies; namely a high number of parents with weight increase compared with weight decrease over time. ${ }^{25}$ 40-42 The average parent was overweight at baseline (with BMI of $29.7 \mathrm{~kg} / \mathrm{m}^{2}$ and $28.8 \mathrm{~kg} / \mathrm{m}^{2}$ in mothers and fathers, respectively) and only $4.3 \%$ mothers and $2.2 \%$ of the fathers decreased their weight by more than $6 \mathrm{~kg}$ during these years. Knowledge of how weight loss in parents may affect offspring weight at a population level is limited, although intervention studies with targeted weight loss have shown parental weight change to be associated with additional weight change in offspring. ${ }^{16} 1743$ Our findings suggest minor healthy changes with regard to maternal weight or physical activity levels during important years of offspring's growth, could positively influence their children's weight development. Hence, mother's position as family caregiver and role model should be considered in future weight management strategies directed towards children and adolescents.

\section{Strengths and limitations}

There are several strengths and weaknesses of the study. A strength is the use of the same protocol for anthropometric measurements in parents and children. Furthermore, not self-reported weight measurements ensured accuracy and circumvented potential bias related to anthropometric measurements. In the weight change analyses, few observations were missing and the main study sample was from an unselected homogeneous population residing in a defined geographical area. The relatively high response rate in both adolescent and parents strengthens the representativeness. That the mean adolescent BMI z-scores in our sample were below 0 may indicate that the adolescents included have lower weight compared with those not included. Still, we do not believe this to have affected the results considerably as a large proportion of the study samples were overweight even so.

The use of education as a proxy of SES in our study, rather than occupation, wealth or deprivation, is based on previous studies which have shown education level to be the measurement variable that best captures inequalities in health. ${ }^{44}$

A limitation of the study is that it only addressed the potential effects of a few obesogenic factors, parental change in weight and physical activity over time. Diet, which is thought to be perhaps the most important obesogenic factor, was not considered in our study due to lack of suitable nutritional data. The reliability and validity of the self-reported physical activity levels may be a limitation in our study. ${ }^{45}$ Improved physical activity measures will be available in the ongoing new wave of the HUNT Study (HUNT4) (data collection 2017-2019) as data are collected through activity censors. Additionally, the physical activity variable used in our study only measures leisure time physical activity. Work-related physical activity may be even more important to take into account these days as an increasing number of occupations require less manual labour and are more sedentary. The unreported work-related physical activity may well be unequally distributed in the two education groups with the low-education group being more work-related physically active than the high education group. This could have led to a bias between the two groups, which have not been considered. Other important factors not accounted for are children's school habits and social relationships. The factors that are focused on, however, are some of the more important ones to consider related to obesity development in a family context. A further limitation is the lack of more data points within the 11-year period considered. Additional data points would have given a more precise estimate of small lifestyle alterations, for example, weight fluctuations or periods of increased physical activity, which could reflect a willingness to alter weight/ lifestyle that could have further affected the offspring.

\section{CONCLUSIONS}

Overall, our study showed an increase in parent's weight and physical activity levels between 1995-1997 and 20062008. In the study period, a maternal weight decrease of 2-6 kg was associated with lower BMI in the adolescent offspring; meanwhile a decrease in physical activity levels in mothers was associated with higher BMI in adolescent offspring. The findings were independent of parental education level, although the effect of weight reduction on adolescents BMI was somewhat more favourable among adolescents with more educated parents. Better knowledge concerning causal mechanisms of obesity development in children, including the impact of lifestyle changes within families, will help healthcare professionals, policy-makers and politicians to improve public obesity prevention strategies.

Acknowledgements The Nord-Trøndelag Health Study (The HUNT Study) is collaboration between HUNT Research Centre (Faculty of Medicine, Norwegian University of Science and Technology NTNU), Nord-Trøndelag Country Council, Central Norway Health Authority and the Norwegian Institute of Public Health.

Contributors KK and TLH initiated the study and acquired the data. TLH was responsible for the Young-HUNT data collection. MN involved in the preparation of the data, the analysis and wrote the first draft of the paper. ERS involved in supervision of statistical analysis and in interpretation of the results. All coauthors critically reviewed during the process, made improvements for the manuscript revisions and approved the final manuscript.

Funding This work was supported by the Liaison Committee between the Norwegian University of Science and Technology and the Central Norway Regional Health Authority, and the NTNU-Norwegian University of Science and Technology, grant number 2013/7511 and the Norwegian Research Council. This research received no specific grant from any funding agency in the public, commercial or not-for-profit sectors.

Disclaimer The funders had no role in study design, data collection and analysis, decision to publish or preparation of the manuscript.

Competing interests None declared.

Patient consent Not required.

Ethics approval Regional Committee for Medical Research Ethics (project no.2013/880, REK Midt, Norway). The protocol was approved by the HUNT Study 
administration, the Regional Committees for Medical and Health Research Ethics and the Norwegian Data Protection Authority.

Provenance and peer review Not commissioned; externally peer reviewed.

Data sharing statement Due to restrictions imposed by the HUNT Research Centre (in accordance with Norwegian Data Inspectorate), data cannot be made publicly available. Data are currently stored in the HUNT databank, and there are restrictions in place for the handling of HUNT data files. Data used from the HUNT Study in research projects will be made available on request to the HUNT Data Access Committee (hunt@medicine.ntnu.no).The HUNT data access information (available here: http://www.ntnu.edu/hunt/data) describes in detail the policy regarding data availability

Open access This is an open access article distributed in accordance with the Creative Commons Attribution Non Commercial (CC BY-NC 4.0) license, which permits others to distribute, remix, adapt, build upon this work non-commercially, and license their derivative works on different terms, provided the original work is properly cited, appropriate credit is given, any changes made indicated, and the use is non-commercial. See: http://creativecommons.org/licenses/by-nc/4.0/.

\section{REFERENCES}

1. Katz DL. Unfattening our children: forks over feet. Int J Obes 2011;35:33-7.

2. Grøholt EK, Stigum H, Nordhagen R. Overweight and obesity among adolescents in Norway: cultural and socio-economic differences. J Public Health 2008;30:258-65.

3. Wangensteen T, Undlien D, Tonstad S, et al. [Genetic causes of obesity]. Tidsskr Nor Laegeforen 2005;125:3090-3.

4. Hill JO, Peters JC. Environmental contributions to the obesity epidemic. Science 1998;280:1371-4.

5. Kalakanis LE, Goldfield GS, Paluch RA, et al. Parental activity as a determinant of activity level and patterns of activity in obese children. Res Q Exerc Sport 2001;72:202-9.

6. Timperio A, Salmon J, Ball K, et al. Family physical activity and sedentary environments and weight change in children. Int $J$ Pediatr Obes 2008;3:160-7.

7. Swinburn BA, Sacks G, Hall KD, et al. The global obesity pandemic: shaped by global drivers and local environments. Lancet 2011;378:804-14.

8. Jeffery RW, Baxter J, McGuire M, et al. Are fast food restaurants an environmental risk factor for obesity? Int J Behav Nutr Phys Act 2006;3:2.

9. Jacques J. Environmental triggers for obesity. OAC News. 2008 http://www.obesityaction.org/wp-content/uploads/EnvironmentalTri ggers.pdf (cited 201726 Oct 2017).

10. Wardle J, Griffith J. Socioeconomic status and weight control practices in British adults. J Epidemiol Community Health 2001;55:185-90.

11. Shrewsbury V, Wardle J. Socioeconomic status and adiposity in childhood: a systematic review of cross-sectional studies 1990-2005. Obesity 2008;16:275-84.

12. Semmler C, Ashcroft J, van Jaarsveld CH, et al. Development of overweight in children in relation to parental weight and socioeconomic status. Obesity 2009;17:814-20.

13. Burke V, Beilin LJ, Dunbar D. Family lifestyle and parental body mass index as predictors of body mass index in Australian children: a longitudinal study. Int J Obes Relat Metab Disord 2001;25:147-57.

14. Jääskeläinen A, Pussinen J, Nuutinen $O$, et al. Intergenerational transmission of overweight among Finnish adolescents and their parents: a 16-year follow-up study. Int J Obes 2011;35:1289-94.

15. Fasting $\mathrm{MH}$, Nilsen TI, Holmen TL, et al. Changes in parental weight and smoking habits and offspring adiposity: data from the HUNTstudy. Int J Pediatr Obes 2011;6:e399-407.

16. Boutelle KN, Cafri G, Crow SJ. Parent predictors of child weight change in family based behavioral obesity treatment. Obesity 2012;20:1539-43.

17. Wrotniak BH, Epstein LH, Paluch RA, et al. Parent weight change as a predictor of child weight change in family-based behavioral obesity treatment. Arch Pediatr Adolesc Med 2004;158:342-7.

18. Jacques-Tiura AJ, Greenwald MK. Behavioral Economic Factors Related to Pediatric Obesity. Pediatr Clin North Am 2016;63:425-46.

19. Ajslev TA, Ängquist L, Silventoinen $\mathrm{K}$, et al. Trends in parent-child correlations of childhood body mass index during the development of the obesity epidemic. PLoS One 2014;9:e109932.

20. Næss M, Holmen TL, Langaas M, et al. Intergenerational transmission of overweight and obesity from parents to their adolescent offspring - the HUNT study. PLoS One 2016;11:e0166585.

21. Li L, Law C, Lo Conte R, et al. Intergenerational influences on childhood body mass index: the effect of parental body mass index trajectories. Am J Clin Nutr 2009;89:551-7.

22. Holmen J. The Nord-Trøndelag Health Study 1995-97 (HUNT 2): objectives, contents, methods and participation. Norsk epidemiologi 2003;13:19-32.

23. Holmen TL, Bratberg G, Krokstad S, et al. Cohort profile of the Young-HUNT Study, Norway: a population-based study of adolescents. Int J Epidemiol 2014;43:536-44.

24. Krokstad S, Langhammer A, Hveem K, et al. Cohort profile: the HUNT Study, Norway. Int J Epidemiol 2013;42:968-77.

25. World Health Organization,. W., Obesity: preventing and managing the global epidemic; report of a WHO consultation. Geneva: World Health Organization, 2000;894:253.

26. Cole TJ, Bellizzi MC, Flegal KM, et al. Establishing a standard definition for child overweight and obesity worldwide: international survey. BMJ 2000;320:1240-3.

27. Kurtze N, Rangul V, Hustvedt BE, et al. Reliability and validity of selfreported physical activity in the Nord-Trøndelag Health Study (HUNT 2). Eur J Epidemiol 2007;22:379-87.

28. Craig CL, Marshall AL, Sjöström M, et al. International physical activity questionnaire: 12 -country reliability and validity. Med Sci Sports Exerc 2003;35:1381-95.

29. SSB. Norwegian standard classification of education (Norsk standard for utdanningsgruppering) Rev. 2000 th ed. Norwegian standard classification of education. Vol. C617. Oslo: Statistics Norway (SSB), 2003.

30. Rabe-Hesketh S, Skrondal A. Multilevel and longitudinal modeling using Stata : Vol. 1: Continuous responses. 3rd ed. College Station, Tex: Stata Press, 2012;1.

31. Ball K, Mishra GD. Whose socioeconomic status influences a woman's obesity risk: her mother's, her father's, or her own? Int J Epidemiol 2006;35:131-8.

32. Mamun AA, Lawlor DA, O'Callaghan MJ, et al. Family and early life factors associated with changes in overweight status between ages 5 and 14 years: findings from the Mater University Study of Pregnancy and its outcomes. Int J Obes 2005;29:475-82.

33. Liu Y, Chen HJ, Liang L, et al. Parent-child resemblance in weight status and its correlates in the United States. PLoS One 2013;8:e65361.

34. Rosenkranz RR, Dzewaltowski DA. Model of the home food environment pertaining to childhood obesity. Nutr Rev 2008;66:123-40.

35. Wang Y, Min J, Khuri J, et al. A Systematic examination of the association between parental and child obesity across countries. Adv Nutr 2017;8:436-48.

36. Greene-Cramer B, Harrell MB, Hoelscher DM, et al. Association between parent and child weight status among private school children in Delhi, India. Glob Health Promot 2018;25:67-74.

37. Bauman A, Ainsworth BE, Sallis JF, et al. The descriptive epidemiology of sitting. A 20-country comparison using the International Physical Activity Questionnaire (IPAQ). Am J Prev Med 2011;41:228-35.

38. Gebel K, Pont S, Ding D, et al. Patterns and predictors of sitting time over ten years in a large population-based Canadian sample: findings from the Canadian Multicentre Osteoporosis Study (CaMos). Prev Med Rep 2017;5:289-94.

39. Davison KK, Birch LL. Obesigenic families: parents' physical activity and dietary intake patterns predict girls' risk of overweight. Int $J$ Obes Relat Metab Disord 2002;26:1186-93.

40. Krokstad S, Ernstsen L, Sund ER, et al. Social and spatial patterns of obesity diffusion over three decades in a Norwegian county population: the HUNT Study. BMC Public Health 2013;13:973.

41. Midthjell K, Lee CM, Langhammer A, et al. Trends in overweight and obesity over 22 years in a large adult population: the HUNT Study, Norway. Clin Obes 2013;3:12-20.

42. Davey Smith G, Steer C, Leary S, et al. Is there an intrauterine influence on obesity? Evidence from parent child associations in the Avon Longitudinal Study of Parents and Children (ALSPAC). Arch Dis Child 2007;92:876-80.

43. Epstein LH, Wing RR, Koeske R, et al. Child and parent weight loss in family-based behavior modification programs. J Consult Clin Psychol 1981;49:674-85.

44. Shavers VL. Measurement of socioeconomic status in health disparities research. J Natl Med Assoc 2007;99:1013-23.

45. Helmerhorst HJ, Brage S, Warren J, et al. A systematic review of reliability and objective criterion-related validity of physical activity questionnaires. Int J Behav Nutr Phys Act 2012;9:103. 